\title{
DIRECTED GRAPHS AND NILPOTENT RINGS
}

\author{
A. V. KELAREV \\ (Received 3 September 1997; revised 10 September 1998)
}

Communicated by J. R. J. Groves

\begin{abstract}
Suppose that a ring is a sum of its nilpotent subrings. We use directed graphs to give new conditions sufficient for the whole ring to be nilpotent.
\end{abstract}

1991 Mathematics subject classification (Amer. Math. Soc.): primary 16N40; secondary 16N60.

The investigation of rings which are sums of their subrings has been carried out by Bahturin and Giambruno [1], Bahturin and Kegel [2], Beidar and Mikhalev [3], Ferrero and Puczyłowski [5], Fukshansky [6], Herstein and Small [7], Kegel [8,9], Kelarev and McConnell [13], Kepczyk and Puczyłowski [14, 15], Puczyłowski [16], Salwa [17] and the author [10-12]. Although there are several positive results which show that some properties are preserved by sums of two subrings, it turns out that relatively few ring-theoretic properties are inherited by rings which are sums of their two subrings, and there are no known nontrivial properties which are inherited by sums of three or more subrings.

A strong negative result of this sort was obtained by Bokut' [4]: Every algebra over a field of characteristic zero can be embedded in a simple algebra which is a sum of three nilpotent subalgebras. In [10] the author constructed a ring which is not nil but is a direct sum of two locally nilpotent subrings. A primitive ring which is a sum of two Wedderburn radical subrings was given in [11] with the use of a homomorphic image of the construction introduced in [10].

Therefore some additional restrictions on the interaction of the summands are needed in order to obtain positive results.

A natural restriction is to require that some products of the subrings are equal to zero. Suppose that a ring $R$ is a sum of its subrings $R_{r}, v \in V$, and assume that for some pairs $u, v \in V$ it is known that the product $R_{u t} R_{\mathrm{r}}$ is equal to zero.

(C) 1998 Australian Mathematical Society 0263-6115/98 \$A2.00+0.00 
We use directed graphs (digraphs) to keep information about all pairs $u, v \in V$ with $R_{u} R_{v}=0$. Denote by $E$ the set of all ordered pairs $u, v \in V$ such that $R_{u} R_{\mathrm{r}}=0$, and consider the digraph $A=(V, E)$. By the complement of $A$ we mean the digraph $G=\bar{A}=(V, \bar{E})$, where $\bar{E}=V \times V \backslash E$. Then we shall say that $R$ is a $G$-sum of the $R_{r}$. The digraph $A$ will be called the annihilator digraph of $R$.

This situation arises in several ring constructions. For example, if $U_{n}(F)$ is the ring of $n \times n$ upper triangular matrices over a field $F$, and $e_{i j}$ denotes the standard matrix unit, then $U_{n}(F)=\oplus_{1 \leq i \leq j \leq n} F e_{i j}$ and $F e_{i j} F e_{k l}=0$ whenever $j \neq k$. For a finite set $V$, a ring $R$ is a direct product of $R_{r}, v \in V$, if and only if $R$ is a $G$-sum, where the annihilator digraph $A=\bar{G}$ is the complete digraph on $V$.

We say that a digraph $G=(V, E)$ is 2-connected if, for any $u, v \in V$, there exists $w \in V \backslash\{u, v\}$ such that $(u, w),(w, v) \in E$.

THEOREM 1. Let $G$ be a digraph without 2-connected subgraphs. If a ring $R$ is a $G$-sum of nilpotent subrings, then $R$ is nilpotent too.

ProOF. Assume that the digraph $G(V, E)$ does not contain any 2-connected subgraphs. Take a ring $R$ which is a $G$-sum of nilpotent subrings $R_{v}, v \in V$. Let $R^{1}$ be the ring $R$ with identity 1 adjoined.

Put $H(R)=\cup_{\mathrm{r} \in V} R_{\imath}$. For any $r \in H(R)$, we fix an element ind $(r) \in V$ such that $r \in R_{\text {ind }(r)}$.

If $U \subseteq V,|U|=k \geq 1$ and $m \geq k$, then by $L(U, m)$ we denote the set of all products of the form $s_{1} t_{l} s_{2} t_{2} \cdots s_{k} t_{k} s_{k+1}$ such that there exist positive integers $a_{1}, \ldots, a_{k}$ satisfying $a_{1}+a_{2}+\cdots+a_{k} \geq m$, where $t_{1}, \ldots, t_{k} \in H(R), t_{l} \in R_{\text {ind }\left(t_{1}\right)}^{a_{1}}$ for $l=1, \ldots, k,\left\{\right.$ ind $\left(t_{1}\right), \ldots$, ind $\left.\left(t_{k}\right)\right\}=U$, and $s_{1}, \ldots, s_{k+1} \in R^{l}$.

For positive integers $k, m, n$, if $k>|V|$, then we put $P(k, m, n)=\{0\}$.

For positive integers $k, m, n$ with $1 \leq k \leq|V|$ and $m \geq k$, denote by $P(k, m, n)$ the set consisting of zero and all products $r_{1} r_{2} \cdots r_{n}$ such that there exists a subset $U \subseteq V$ satisfying $|U|=k$ and $r_{1}, \ldots, r_{n} \in L(U, m)$.

We claim that every product in $P(k, m, 3(|V|+1) n)$ is a sum of elements from $P(k, m+1, n)$ and $P(k+1, m+1, n)$.

For $k>|V|$ the assertion is trivial. Assume that $k \leq|V|$. Take any product $w=$ $r_{1} r_{2} \cdots r_{3|V|+1) n} \in P(k, m, 3(|V|+1) n)$. By the definition of $P(k, m, 3(|V|+1) n)$ there exists a subset $U \subseteq V$ such that $|U|=k$ and $r_{1}, \ldots, r_{3(|V|+\mid) n} \in L(U, m)$.

For any $i=0,1, \ldots,(|V|+1) n-1$, we rewrite the elements $r_{3 i+1}, r_{3 i+2}, r_{3 i+3}$ and introduce an auxiliary set $w_{i}$ which characterizes the way we rewrite them.

The definition of $L(U, m)$ shows that $r_{3 i+j}=s_{j .1} t_{j .1} s_{j .2} t_{j .2} \cdots s_{j . k} t_{j . k} s_{j . k+1}$, for $j=1,2,3$, where there exist positive integers $a_{j .1}, \ldots, a_{j . k}$ such that

$$
a_{j .1}+a_{j .2}+\cdots+a_{j . k} \geq m
$$


$t_{j, 1}, \ldots, t_{j, k} \in H(R), t_{j . l} \in R_{\text {ind }\left(t_{i, l}\right)}^{a_{j, l}}$ for $l=1, \ldots, k,\left\{\operatorname{ind}\left(t_{j, 1}\right) \ldots, \operatorname{ind}\left(t_{j . k}\right)\right\}=U$, and $s_{j .1}, \ldots, s_{j . k+1} \in R^{1}$.

If the three sets of pairs

$$
\left\{\left(\operatorname{ind}\left(t_{j, 1}\right), a_{j .1}\right), \ldots,\left(\text { ind }\left(t_{j, k}\right), a_{j . k}\right)\right\}, \quad j=1,2,3
$$

are not equal to each other, then for each value of ind $\left(t_{j .1}\right)$ we can choose the maximum power $a_{j . l}$ out of the three available powers $a_{j . l}, j=1,2,3$. Since for some ind $\left(t_{j . l}\right)$ the elements $a_{j, l}, j=1,2,3$ are not all equal, it follows that the sum of exponents of the chosen maximal powers is strictly greater than $m$. We keep the corresponding maximal elements $t_{j . l} \in R_{\text {ind }\left(t_{, j,}\right)}^{a_{j, l}}$ and multiply together the other elements which are between them. In this way we rewrite $r_{3 i+1} r_{3 i+2} r_{3 i+3}$ as a product in $L(U, m+1)$. In this case we put $w_{i}=\emptyset$ to remember that $r_{3 i+1} r_{3 i+2} r_{3 i+3}$ has been rewritten as a product in $L(U, m+1)$.

Next consider the case where all three sets of pairs

$$
\left\{\left(\operatorname{ind}\left(t_{j .1}\right), a_{j .1}\right), \ldots,\left(\text { ind }\left(t_{j, k}\right), a_{j . k}\right)\right\}, \quad j=1,2,3
$$

are equal to each other. In this case we rewrite $r_{3 i+1} r_{3 i+2} r_{3 i+3}$ as a sum of several elements, we consider only one summand and we introduce $u_{i}$ to characterize this summand.

Given that the graph $G(V, E)$ is not 2-connected, we can find $u_{1}, u_{2} \in U$ such that for any $w \in U \backslash\left\{u_{1}, u_{2}\right\}$ either $\left(u_{1}, w\right) \notin E$ or $\left(w, u_{2}\right) \notin E$. Then we can find $l_{1}, l_{2}$ such that ind $\left(t_{2, l_{1}}\right)=u_{1}$ and $\operatorname{ind}\left(t_{3, l_{2}}\right)=u_{2}$. Let $r_{3 i+2}=a_{1} t_{2, l_{1}}^{a_{2, l_{1}}} b_{1}$ and $r_{3 i+3}=a_{2} t_{3, l_{2}}^{a_{3, l_{2}}} b_{2}$. Multiplying together $b_{1} a_{2}$ we use the fact that $R=\bigoplus_{v \in l}, R_{v^{\prime}}$ and represent the product as a sum $b_{1} a_{2}=\sum_{v \in V} c_{v}$, where $c_{v} \in R_{v}$. When we substitute the sum for $b_{1} a_{2}$, the product $r_{3 i+1} r_{3 i+2} r_{3 i+3}$ turns into a sum of several elements $r_{3 i+1} a_{1} t_{2 . l_{1}} c_{1} t_{3, I_{2}} b_{2}$, where $v \in V$. We consider only one of these elements, for an arbitrary $v \in V$. Naturally, the product $r_{1} \cdots r_{3(|V|+1) n}$ also becomes a sum of several summands, and we consider only one of these summands.

If $v=u_{1}$, then $t_{2 . l_{1}} c_{v^{\prime}} \in R_{u_{1}}^{a_{2 \prime_{1}}+1}$. Using this we can rewrite $r_{3 i+1} r_{3 i+2} r_{3 i+3}$ as a product in $L(U, m+1)$ and we put $w_{i}=\emptyset$.

If $v=u_{2}$, then $c_{v} t_{3 . l_{2}} \in R_{u_{2}}^{a_{3, l_{2}+1}}$. Using this we can rewrite $r_{3 i+1} r_{3 i+2} r_{3 i+3}$ as a product in $L(U, m+1)$ and we put $w_{i}=\emptyset$.

If $v \in U \backslash\left\{u_{1}, u_{2}\right\}$, then either $\left(u_{1}, v\right) \notin E$ or $\left(v, u_{2}\right) \notin E$. It follows that either $t_{2, l_{1}} c_{2^{\prime}}=0$ or $c_{2} t_{3 . l_{2}}=0$, respectively. Therefore $r_{3 i+1} a_{1} t_{2, l_{1}} c_{2^{\prime}} t_{3 l_{2} l_{2}} b_{2}=0$. In this case the corresponding summand of $r_{1} \cdots r_{3(|V|+\mid) n}$ is zero and belongs to $P(k, m+1, n)$, as claimed.

If $v \in V \backslash U$, then we rewrite $r_{3 i+1} r_{3 i+2} r_{3 i+3}$ as a product in $L(U \cup\{v\}, m+1)$ and we put $w_{i}=\left\{c_{r}\right\}$.

Thus all products $r_{3 i+1} r_{3 i+2} r_{3 i+3}$ have been rewritten. Therefore the whole product $r_{1} \cdots r_{3|v|+1 \text { in }}$ has also been rewritten. We consider only one summand $s$ of 
$r_{1} \ldots r_{3\left(i, v_{\mid+1}\right)}$. The corresponding elements $w_{1}, \ldots, w_{\{|V|+1)}$ characterizing this summand $s$ have been introduced.

Since the elements $w_{1}, \ldots, w_{\{|V|+1) n}$ are chosen in $V \cup\{\emptyset\}$, there exist

$$
1 \leq i_{1}<\cdots<i_{n} \leq(|V|+1) n
$$

such that $w_{i_{1}}=\cdots=w_{i_{n}}=w$.

If $w=\emptyset$, then all the summands of $r_{3 i_{i}+1} r_{3 i_{+}+2} r_{3 i_{i}+3}, l=1, \ldots, n$, which we considered, have been rewritten as elements of $L(U, m+1)$. Therefore we can rewrite the whole summand $s$ as an element of $P(k, m+1, n)$, as claimed.

If $w=\{v\}$ for $v \in V$, then all the summands of $r_{3 i_{i}+1} r_{3 i_{+}+2} r_{3 i_{l}+3}, l=1, \ldots n$, which we considered, have been rewritten as elements of $L(U \cup\{v\}, m+1)$. Therefore we can rewrite the whole summand $s$ as an element of $P(k, m+1, n)$, as claimed.

Thus every product in $P(k, m, 3(|V|+1) n)$ is a sum of elements from $P(k, m+1, n)$ and $P(k+1, m+1, n)$.

Denote by $N$ the maximum of the nilpotency indices of the rings $R_{v}, v \in V$. Then $R_{v}^{N}=0$ for all $v$. Easy induction shows that every product in

$$
P\left(1,1,[3(|V|+1)]^{N|V|}\right)
$$

is a sum of elements from the sets $P(k, 1+N|V|, 1)$, for $1 \leq k \leq|V|$.

Take any element $r$ in $P(k, 1+N|V|, 1)$. By the definition there exists a subset $U \subseteq V$ such that $|U|=k$ and $r \in L(U, 1+N|V|)$. Therefore $r=s_{1} t_{1} s_{2} t_{2} \cdots s_{k} t_{k} s_{k+1}$ and there exist positive integers $a_{1} \ldots, a_{k}$ satisfying $a_{1}+a_{2}+\cdots+a_{k} \geq 1+N|V|$, where $t_{1}, \ldots, t_{k} \in H(R), t_{l} \in R_{\text {ind }\left(t_{l}\right)}^{a_{l}}$ for $l=1, \ldots, k,\left\{\operatorname{ind}\left(t_{1}\right), \ldots, \operatorname{ind}\left(t_{k}\right)\right\}=U$, and $s_{1}, \ldots, s_{k+1} \in R^{1}$. We can choose a maximum exponent $a_{i}$ for some $1 \leq i \leq k$. Clearly, $a_{i} \geq N$, and so $t_{i} \in R_{\left.\text {ind } t_{i}\right)}^{a_{t}}=0$. It follows that $r=0$.

Thus $P(k, 1+N|V|, 1)=\{0\}$. Therefore $P\left(1,1,[3(|V|+1)]^{N|V|}\right)=0$.

Put $n=|V|\left\{[3(|V|+1)]^{N|V|}-1\right\}+1$, and consider an arbitrary product $w=$ $r_{1} \cdots r_{n}$, where $r_{1}, \ldots, r_{n} \in H(R)$. Since ind $\left(r_{i}\right) \in V$ for all $i$, clearly there exist numbers

$$
1 \leq i_{1}<i_{2}<\cdots<i_{[3(|V|+1)]^{*}} \leq|V|\left\{[3(|V|+1)]^{N|V|}-1\right\}+1
$$

such that

$$
\operatorname{ind}\left(r_{i_{1}}\right)=\operatorname{ind}\left(r_{i_{2}}\right)=\cdots=\operatorname{ind}\left(r_{i_{13,1+1,1} v}\right)=v .
$$

Every element $r_{i}$, belongs to $L(\{v\}, 1)$. Therefore $w$ can be rewritten as a product in $P\left(1,1,[3(|V|+1)]^{N|V|}\right)=0$. Thus $H(R)^{n}=0$, and so $R^{n}=0$.

COROLLARY 2. For a graph $G=(V, E)$ the following conditions are equivalent: 
(i) if a ring $R$ is a $G$-sum of nilpotent subrings, then $R$ is nilpotent too;

(ii) $G$ does not contain triangles.

ProOF. (i) $\Rightarrow$ (ii): Suppose that (ii) is not satisfied, that is $G$ contains a triangle. Then Bokut's example of a ring which is not nilpotent but is a sum of three nilpotent subrings can be easily made a $G$-sum of the three nilpotent subrings and several zero subrings. Thus (i) does not hold. Thus (i) implies (ii).

(ii) $\Rightarrow$ (i): We can view the graph $G$ as a digraph associating with every undirected edge two directed edges. Then it is easily seen that every 2 -connected graph contains a triangle. Thus $G$ does not contain 2 -connected subgraphs by (ii). Theorem 1 yields (i).

There exist directed graphs which are 2-connected but contain no triangles. For example, take $G=(V, E)$ with $V=\left\{O, A_{1}, \ldots, A_{n}\right\}$, where $O$ is connected to all $A_{1}, \ldots, A_{n}$ by two-sided edges, each $A_{i}$ is connected to $A_{i+1}$ and $A_{n}$ is connected to $A_{1}$ by directed edges.

Next, we discuss an example which shows that our Theorem 1 is probably not improvable. Let $G=(V, E)$ be a digraph containing a 2-connected digraph $H=$ ( $W, \bar{F}$ ) where $W \subseteq V, F \subseteq E$. We define a ring $R$ which is an $H$-sum of subrings $R_{u}, w \in W$, with zero multiplication. If, after that, we put $R_{r}=0$ for all $v \in V \backslash W$, then we see that $R$ is a $G$-sum of the $R_{r}$. Hence we may throw out the vertices of $G$ which do not belong to the 2-connected digraph $H$ and assume that $G$ is 2-connected from the very beginning. We also assume that $E$ contains no loop $(v, v)$, since we can throw away all loops from $E$ without changing the 2-connectedness of $G$. Let $n=|V|$. To simplify further notation we assume that $V=\{1, \ldots, n\}$.

Let $M$ be the set of terms formed by variables $x_{1}, \ldots, x_{n}$ with respect to $n$ nonassociative operations $f_{1}, \ldots, f_{n}$. It can be defined recursively by the following two conditions:

(i) $x_{1}, \ldots, x_{n} \in M$

(ii) $f_{i}(y, z)$ for all $y, z \in M$ and $i \in\{1, \ldots, n\}$.

For $i=1, \ldots, n$, we define the sets

$$
M_{i}=\left\{x_{i}\right\} \cup\left\{f_{i}(y, z) \mid y, z \in M\right\} .
$$

Then $M=M_{1} \cup \cdots \cup M_{n}$. For any $y \in M$, there exists an integer ind( $(y)$ such that $y \in M_{\text {ind }(y)}$.

Let $\mathbb{R}$ be the field of real numbers. We define an $\mathbb{R}$-algebra $R$ generated by the set $M$ subject to relations

$$
y z-f_{1}(y, z)-\cdots-f_{n}(y, z)=0
$$


for all $y, z \in M$ such that (ind $(y)$, ind $(z)) \in E$;

$$
u v=f_{1}(u, v)=\cdots=f_{n}(u, v)=0
$$

for all $u, v \in M$ such that $(\operatorname{ind}(u)$, ind $(v)) \notin E$.

For $i=1, \ldots, n$, denote by $R_{i}$ the subspace spanned over $\mathbb{R}$ by $M_{i}$. The relations (1) and (2) show that $R=\sum_{i=1}^{n} R_{n}$ is a $G$-sum. Given that $E$ contains no loops $(v, v)$, $v \in V$, it follows from (2) that all $R_{1}, \ldots, R_{n}$ are rings with zero multiplication.

Obviously, every 2 -connected graph contains a directed cycle. Let $i_{1}, \ldots, i_{k}, i_{1}$ be a directed cycle in $G$. Then it seems that $w=\left(x_{i_{1}} \cdots x_{i_{h}}\right)^{m}$ is nonzero for all positive integers $m$. The diamond lemma suggests itself as a tool for proving this.

In conclusion we look at the ring $S U_{n}(R)$ of strictly upper triangular matrices over any ring $R$ to illustrate Theorem 1. Clearly, $S U_{n}(R)=\sum_{i<j} R e_{i j}$, where $e_{i j}$ is the standard matrix unit. All the rings $R e_{i j}$ have zero multiplication for $1 \leq i<j \leq n$. If we put $G=(V, E)$, where $V=\{(i, j) \mid 1 \leq i<j \leq n\}$ and $E=\{((i, j),(j, k))$ $1 \leq i<j<k \leq n\}$, then we see that $S U_{n}(R)$ is a $G$-sum of the rings $R e_{i j}$. It follows from Theorem 1 that $S U_{n}(R)$ is nilpotent.

\section{References}

[1] Yu. A. Bahturin and A. Giambruno, 'Identities of sums of commutative subalgebras' Rend. Circ. Mat. Palermo (2) 43 (1994)(2), 250-258.

[2] Yu. A. Bahturin and O. H. Kegel, 'Lie algebras which are universal sums of abelian subalgebras', Comm. Algebra 23 (1995), 2975-2990.

[3] K. I. Beidar and A. V. Mikhalev, 'Generalized polynomial identities and rings which are sums of two subrings', Algebra i Logika 34 (1995)(1), 3-11.

[4] L. A. Bokut', 'Embeddings in simple associative algebras', Algebra i Logika 15 (1976)(2), 117-142.

[5] M. Ferrero and E. R. Puczyłowski, 'On rings which are sums of two subrings', Arch. Math. (Basel) 53 (1989), 4-10.

[6] A. Fukshansky, 'The sum of two locally nilpotent rings may contain a non-commutative free subring', Proc. Amer. Math. Soc, to appear.

[7] I. N. Herstein and L. W. Small, 'Nil rings satisfying certain chain conditions', Canad. J. Math. 16 (1964), 771-776.

[8] O. H. Kegel, 'Zur Nilpotenz gewisser assoziativer Ringe', Math. Ann. 149 (1962/63), 258-260.

[9] O. H. Kegel, 'On rings that are sums of two subrings', J. Algebra 1 (1964), 103-109.

[10] A. V. Kelarev, 'A sum of two locally nilpotent rings may be not nil', Arch. Math. (Basel) 60 (1993), $431-435$.

[11] A. V. Kelarev, 'A primitive ring which is a sum of two Wedderburn radical subrings', Proc. Amer. Math. Soc. 125 (1997), 2191-2193.

[12] A. V. Kelarev, 'An answer to a question of Kegel on sums of rings', Canad. Math. Bull. 41 (1998), 79-80.

[13] A. V. Kelarev and N. R. McConnell, 'Two versions of graded rings', Publ. Math. (Debrecen) 47 (1995) (3-4), 219-227. 
[14] M. Kepczyk and E.R. Puczylowski, 'On radicals of rings which are sums of two subrings', Arch. Math. (Basel) 66 (1996), 8-12.

[15] M. Kepczyk and E.R. Puczyłowski, 'Rings which are sums of two subrings'. J. Pure Appl. Algebra. to appear.

[16] E. R. Puczyłowski, 'Some questions concerning radicals of associative rings', Theory of Radicals. Szekszárd, 1991, Coll. Math. Soc. János Bolyai 61 (1993), $209-227$.

[17] A. Salwa, 'Rings that are sums of two locally nilpotent subrings'. Comm. Algebra 24 (1996)(12), 3921-3931.

School of Mathematics

University of Tasmania

G.P.O. Box 252-37

Hobart, Tasmania 7001

Australia

e-mail: kelarev@hilbert.maths.utas.edu.au 